Management international

International Management

Gestiòn Internacional

Discours médiatique sur les activités des multinationales à

l'étranger : Le cas de Danone en Chine

Media Discourse on Multinational Companies' Activities

Overseas: The Case of Danone in China

Discurso mediático sobre las actividades internacionales de las

empresas multinacionales: el caso de Danone en China

François Goxe et Michaël Viegas Pires

Volume 20, numéro 3, printemps 2016

URI : https://id.erudit.org/iderudit/1051309ar

DOI : https://doi.org/10.7202/1051309ar

Aller au sommaire du numéro

Éditeur(s)

HEC Montréal

Université Paris Dauphine

ISSN

1206-1697 (imprimé)

1918-9222 (numérique)

Découvrir la revue

Citer cet article

Goxe, F. \& Viegas Pires, M. (2016). Discours médiatique sur les activités des multinationales à l'étranger : Le cas de Danone en Chine. Management international / International Management / Gestiòn Internacional, 20(3), 90-104. https://doi.org/10.7202/1051309ar
Résumé de l'article

Cet article s'intéresse à la façon dont les activités de firmes multinationales (FMN) à l'étranger sont présentées dans les média de leur pays d'origine. Il s'appuie sur le cas de Danone en Chine, traité à partir de données tirées de la presse économique en recourant à l'analyse critique du discours. Il montre comment le propos médiatique s'articule autour de quelques discours types, et principalement un discours culturel/nationaliste. Il questionne ainsi la portée des schémas discursifs déjà identifiés dans la littérature, en même temps qu'il les complète.
Tous droits réservés @ Management international / International Management / Gestión Internacional, 2016
Ce document est protégé par la loi sur le droit d'auteur. L’utilisation des services d’Érudit (y compris la reproduction) est assujettie à sa politique d'utilisation que vous pouvez consulter en ligne.

https://apropos.erudit.org/fr/usagers/politique-dutilisation/ 


\title{
Discours médiatique sur les activités des multinationales à l'étranger : Le cas de Danone en Chine
}

\author{
Media Discourse on Multinational Companies' \\ Activities Overseas : The Case of Danone in China
}

\section{Discurso mediático sobre las actividades internacionales de las empresas multinacionales : el caso de Danone en China}

\author{
FRANCOIS GOXE \\ Université de Versailles St Quentin, \\ ISM-LAREQUOI
}

\section{RÉSUMÉ}

Cet article s'intéresse à la façon dont les activités de firmes multinationales (FMN) à l'étranger sont présentées dans les média de leur pays d'origine. Il s'appuie sur le cas de Danone en Chine, traité à partir de données tirées de la presse économique en recourant à l'analyse critique du discours. Il montre comment le propos médiatique s'articule autour de quelques discours types, et principalement un discours culturel/nationaliste. Il questionne ainsi la portée des schémas discursifs déjà identifiés dans la littérature, en même temps qu'il les complète.

Mots clés : discours, analyse critique du discours, firmes multinationales, média

\author{
MICHAËL VIEGAS PIRES \\ Université de Versailles St Quentin, \\ ISM-LAREQUOI
}

\section{ABSTRACT}

This article looks at how the activities of multinational companies (MNCs) abroad are presented in the media of their country of origin. Drawing on the critical discourse analysis of business press articles related to the operations of the French multinational Danone in China, it reveals how the media's speech revolves around some typical types of discourse, mainly a cultural / nationalist one. The article thus questions the scope of discursive patterns already identified in the literature, while it complements them.

Keywords: discourse, critical discourse analysis, multinationals, media

\begin{abstract}
RESUMEN
En este artículo, se analiza cómo las actividades internacionales de las empresas multinacionales (EMN) están presentadas en los medios de comunicación de su país de origen. Se basa en el caso de Danone en China, tratado a partir de datos de la prensa de negocios mediante el análisis crítico del discurso. Se muestra cómo el discurso de los medios de comunicación giran en torno a algunos discursos típicos, y sobre todo a un discurso cultural / nacionalista. Por lo tanto, el articulo cuestiona el alcance de los patrones discursivos ya identificados en la literatura mientras que los complementa.
\end{abstract}

Palabras Claves: discurso, análisis crítico del discurso, empresas multinacionales, medios de comunicación
$\mathrm{Q}$ u'il s'agisse de s'accorder sur un diagnostic, de discuter d'opportunités, ou bien même de communiquer la stratégie, il n'est de pratique stratégique qui ne recourt au langage. Plus qu'un simple outil, le discours participe de la construction sociale de la réalité organisationnelle (Fairclough, 2005). Il est ce qui donne une existence sociale à des objets tels que les opportunités, les marchés ou encore les compétences (Ezzamel et Willmott, 2008). Lapproche discursive de la stratégie, qui cherche à comprendre en quoi le langage façonne la stratégie et réciproquement, est donc un prisme particulièrement fécond pour la recherche en management stratégique (Balogun et al., 2014; Phillips et al., 2008).

Les recherches s'inscrivant dans cette approche ont notamment montré que le discours joue un rôle central dans les processus stratégiques de sensemaking et sensegiving (Gioia et Chittipeddi, 1991). Le discours influence en effet la façon dont les managers interprètent l'information stratégique (sensemaking) en donnant un cadre à leurs représentations mentales. Il est également un moyen par lequel les managers cherchent à influencer le sens donné par les autres membres de lorganisation à la stratégie et ses fondements (sensegiving). Adoptant tour à tour une perspective narrative (Barry et Elmes, 1997; Vaara, 2002), rhétorique (Chanal et Tannery, 2005, 2007), ou conversationnelle (Rouleau, 2005; SamraFredericks, 2003), de multiples recherches se sont ainsi attachées à comprendre les pratiques discursives liées à la «mise en sens ${ }^{1}$ de la stratégie.

Ces recherches ont en commun de rester focalisées sur le rôle des managers. Cette mise en sens est pourtant également le fait d'autres acteurs, notamment extérieurs à l'organisation. Certains travaux suggèrent en particulier, que les média tels que la presse économique et grand public jouent un

1. Par commodité, nous utilisons ici le terme générique de «mise en sens» pour désigner à la fois les processus de sensemaking et sensegiving. 
rôle important dans ces processus. En privilégiant certaines interprétations des faits au détriment d'autres (Hellgren et al., 2002), les média participent à la construction sociale de la réalité organisationnelle (Risberg et al., 2003; Tienari et al., 2003). L'interprétation de la stratégie par les média reste cependant un domaine encore relativement peu exploré, et les quelques travaux sur le sujet concernent le seul champ des fusions-acquisitions (F\&A) (Kuronen et al., 2005; Risberg et al., 2003; Vaara et Tienari, 2002; Zhu et McKenna, 2012). La présente recherche a donc pour objectif d'approfondir et d'élargir l'analyse en s'intéressant au discours médiatique sur l'ensemble des activités des firmes multinationales (FMN), et en particulier celles menées à létranger.

Les activités internationales des FMN sont un contexte particulièrement pertinent pour étudier la construction et la diffusion de sens autour de la stratégie par les média. En effet, ces activités sont par nature multidimensionnelles et peuvent renvoyer à de multiples sujets tels que les modalités d'internationalisation, l'impact économique des FMN ou encore les succès et les échecs dans les stratégies d'internationalisation. Chaque sujet peut lui même donner lieu à différentes lectures, en termes de pertinence stratégique, d'interactions interculturelles, ou encore de relations politico-diplomatiques entre les pays concernés. Il est donc particulièrement intéressant d'observer si certains sujets et/ou interprétations dominent le discours médiatique, et d'en discuter les raisons. D’une manière générale, la mise en sens des activités des FMN à l'étranger dans les média est un sujet peu étudié. Si quelques travaux se sont intéressés aux pratiques de légitimation discursive des activités internationales des FMN, celles-ci sont le plus souvent appréhendées seulement du point de vue de l'entreprise, notamment à la lumière de sa communication institutionnelle (Sergot et Claret, 2011).

Cette recherche sappuie sur le cas de Danone en Chine, traité à partir de données tirées de la presse économique française, et en recourant à l'analyse critique du discours (Fairclough, 2005). Reprenant les travaux sur la légitimation discursive des décisions stratégiques (Sergot et Claret, 2011; Tienari et al., 2003; Vaara et Tienari, 2002,2008) et les différents discours types qu'ils ont permis d'identifier (Balogun et al., 2011; Vaara et Tienari, 2002, 2008), elle analyse la façon dont les activités d'une FMN à l'international sont interprétées par les média de son pays dorigine. Elle pose donc la question suivante : comment les média domestiques font-ils sens des activités menées par les firmes multinationales (FMN) dans leurs marchés hôtes?

Au-delà de la simple étude d'un contexte nouveau, cette recherche questionne ainsi la portée des schémas discursifs types identifiés dans la littérature sur les F\&A (Vaara, 2002; Vaara et Tienari, 2002), en montrant que les média privilégient d'autres formes de discours lorsqu'il s'agit de rendre compte du développement international d'une FMN. Elle suggère ainsi que les discours types que mobilisent les média dépendent des évènements qu'ils traitent et de leur contexte.

Larticle est structuré comme suit. Une première partie présente le cadre théorique de la recherche, en donnant un aperçu des recherches en analyse de discours. Une deuxième partie présente la méthodologie de recherche. Les résultats sont présentés dans une troisième partie et l'article se conclut par une discussion des contributions et implications.

\section{Cadre théorique}

\section{SENSEMAKING, SENSEGIVING, ET DISCOURS MÉDIATIQUE}

Les concepts de sensemaking et sensegiving renvoient à deux processus complémentaires et interdépendants. Alors que le sensemaking correspond à l'interprétation de l'information stratégique par les managers pour eux-mêmes, le sensegiving est le processus par lequel ces derniers cherchent à influencer l'interprétation des autres membres de l'organisation (Gioia et Chittipeddi, 1991). Le discours joue un rôle central dans ces deux processus. Dans le contexte du sensemaking, il donne un cadre à nos représentations mentales du monde (Paroutis et Heracleous, 2013). Il influence donc la façon dont les managers interprètent l'information stratégique. Différentes recherches montrent également que le sensegiving requiert que les managers fassent preuve de certaines compétences discursives (Maitlis et Lawrence, 2007; Rouleau et Balogun, 2011). Létude des pratiques discursives au centre du processus de sensegiving a ainsi donné lieu à de nombreuses recherches. Celles-ci ont exploré les processus conversationnels (Rouleau, 2005; Samra-Fredericks, 2003), narratifs (Barry et Elmes, 1997; Vaara, 2002) ou rhétoriques (Chanal et Tannery, 2005, 2007) par lesquels les managers cherchent à influencer les interprétations des autres acteurs organisationnels dans un contexte de changement stratégique.

A ce jour, les travaux sur la mise en sens de la stratégie se sont donc essentiellement focalisés sur les pratiques discursives des managers (Balogun et al., 2014) bien que d'autres acteurs y participent. Les média semblent en particulier jouer un rôle important. Les évènements qui marquent la vie des entreprises peuvent être sujets à de multiples interprétations. Les média mettent en avant certaines de ces interprétations, tout en marginalisant d'autres «réalités » possibles (Hellgren et al., 2002, p. 124). Par exemple, Vaara et Tienari (2002) montrent, en étudiant quatre cas de F\&A en Finlande, que le discours médiatique met l'accent sur les logiques stratégiques et managériales de ces opérations tout en marginalisant d'autres sujets comme leur impact sociétal, notamment en termes d'emploi.

Les média participent donc à la construction sociale de la réalité (Risberg et al., 2003; Tienari et al., 2003). Ils occupent une place centrale dans les sociétés modernes, reflétant la vision qu'a le public de différents sujets tout en l'influençant (Kuronen et al., 2005). En d'autres termes, les média peuvent être considérés à la fois comme "sensemaker» et "sense-giver» (Hellgren et al., 2002, p. 123). Le discours médiatique est un cadre par lequel les journalistes font sens de la réalité organisationnelle (sensemaking). Dans le même temps, les versions de la réalité qu'ils promeuvent dans ce discours peuvent influencer la perception du public (sensegiving) (Risberg et al., 2003). La presse "grand public» est ainsi, par exemple, un lieu important pour la diffusion 
et la reproduction de la légitimité des idées et pratiques managériales en général (Abrahamson, 1996; Mazza et Alvarez, 2000). Dans le contexte de F\&A en particulier (Vaara et Tienari, 2002; Zhu et McKenna, 2012), la presse peut, par exemple, mettre laccent sur le besoin de restructuration et le développement de l'avantage concurrentiel permis par la fusion et ainsi convaincre le lecteur des bienfaits de ce mouvement stratégique. $\mathrm{Si}$, au contraire, la presse met l'accent sur les pertes d'emplois et le sort des employés licenciés, l'image véhiculée est plus négative et contrarie la légitimité de l'entreprise.

\section{L'ANALYSE CRITIQUE DU DISCOURS}

Au sein des différentes perspectives constitutives de l'approche discursive de la stratégie, cette recherche s'inscrit plus particulièrement dans celle de l'Analyse Critique du Discours (désormais ACD). Dans la mouvance des travaux de Vaara et de ses collègues (Tienari et al., 2003; Vaara, 2002; Vaara et Tienari, 2002, 2008), nous concevons l'ACD comme «une méthode pour analyser la construction sociale de phénomènes organisationnels sous une forme textuelle» (Vaara et Tienari, 2002, p. 4).

L'ACD s'inscrit dans la tradition de la linguistique, et implique une analyse détaillée du texte ${ }^{2}$ (Balogun et al., 2014; Vaara et Tienari, 2008). Elle suppose que le discours ne rend pas objectivement compte d'une réalité organisationnelle mais participe de sa construction sociale (Fairclough, 2005). Il (re)définit ce qui est justifié et légitime (Tienari et al., 2003; Vaara et Tienari, 2002) et peut de ce fait être mobilisé comme une ressource dans les processus de développement et de changement stratégiques (Balogun et al., 2014).

L'ACD s'efforce également de resituer la construction textuelle des phénomènes organisationnels dans un contexte social plus large (Leitch et Palmer, 2010). Ce contexte est animé par diverses relations de pouvoir, à l'aune desquelles le discours organisationnel est à interpréter. C'est en ce sens que l'ACD adopte un positionnement critique. Elle s'attache à comprendre le rôle du discours dans la (re)production des relations de pouvoir et des structures de domination (van Dijk, 1993). Dans le cas de multinationales, ces relations de pouvoir peuvent renvoyer à des rapports entre Etats, entre organisations ou même entre individus. Par exemple, Risberg et al. (2003) montrent comment, dans le cadre d'une acquisition transfrontalière, les relations historiques entre la Suède et la Norvège se sont traduites par un affrontement à caractère nationaliste dans les discussions publiques autour de l'évènement.

\section{DES TEXTES DOMINÉS PAR UN DISCOURS RATIONALISTE}

Les travaux mobilisant l'ACD dans le champ de la stratégie, et plus particulièrement au sujet des F\&A, ont permis d'identifier différents discours types imbriqués dans le texte, selon lesquels les activités et manœuvres stratégiques des entreprises sont le plus souvent restituées. Chaque discours fait référence à des thèmes spécifiques et se focalise sur différents éléments. Le Tableau 1 offre une vue synthétique des principaux discours issus de ces travaux.

Le discours le plus fréquemment mobilisé est un discours dit « rationaliste», centré sur l'entreprise, les différents moyens que celle-ci et ses acteurs mettent en œuvre pour développer sa compétitivité et défendre ou obtenir un avantage concurrentiel et les raisons «rationnelles» de ces actions. Ce discours mobilise largement le jargon du management stratégique. Le discours «culturel», quant à lui, se focalise sur les cultures et sous-cultures et les affrontements culturels potentiels entre acteurs, individus ou entreprises. Dans une variante «nationaliste», il met plus particulièrement l'accent sur les différences nationales, et est fréquemment mobilisé dans le contexte de F\&A transfrontalières qui sont alors interprétées comme une confrontation entre différents camps nationaux (Hellgren et al., 2002; Risberg et al., 2003; Tienari et al., 2003). Dans ce discours, les organisations et les individus sont souvent identifiés par leur origine géoculturelle et par différentes idées ou symboles qui définissent cette dernière (Riad et Vaara, 2011). Enfin, le discours «individualiste» se concentre sur les individus, leurs personnalités et caractéristiques (Vaara, 2002). Il personnifie les actions des entreprises, en particulier sous les traits de leurs dirigeants et/ ou managers.

D’autres discours ont été identifiés dans la littérature,

\begin{tabular}{|c|c|c|}
\hline \multicolumn{3}{|c|}{$\begin{array}{l}\text { TABLEAU } 1 \\
\text { Principaux discours types }\end{array}$} \\
\hline Discours & Focus & Références (exemples) \\
\hline Rationaliste & Entreprise, organisation, compétitivité & $\begin{array}{l}\text { (Balogun et al., 2011; Hellgren et al., 2002; } \\
\text { Kuronen et al., 2005; Vaara, 2002; Vaara et } \\
\text { Tienari, 2002) }\end{array}$ \\
\hline Culturel/Nationaliste & $\begin{array}{l}\text { Cultures, différences culturelles, } \\
\text { affrontements culturels, différences } \\
\text { nationales }\end{array}$ & $\begin{array}{l}\text { (Balogun et al., 2011; Hellgren et al., 2002; } \\
\text { Risberg et al., 2003; Tienari et al., 2003) }\end{array}$ \\
\hline Individualiste & Individus, personnalités & (Vaara, 2002; Vaara et Tienari, 2002) \\
\hline
\end{tabular}

2. Les textes sont des instances concrètes ou unités fondamentales du discours, lui-même conçu à la fois comme un ensemble de textes et une activité sociale visant à donner du sens (Phillips et al., 2008). Il ne s'agit pas seulement de textes écrits, mais de toute forme d'utilisation du langage tels que des conversations, des présentations orales, etc., au travers de différents média écrits, visuels, auditifs ou audiovisuels. 
quoique mobilisés de façon plus marginale. Il s’agit par exemple du discours «sociétal» qui s'intéresse aux conséquences sociales et sociétales des activités des entreprises (Vaara et Tienari, 2002), ou encore de discours centrés sur la position des acteurs dans la hiérarchie (Vaara, 2002).

Notre propos n'est pas ici de recenser de manière exhaustive ces discours types, mais plus simplement de rappeler que les discussions autour des manœuvres stratégiques des entreprises sarticulent autour de quelques schémas discursifs types, et sont plus particulièrement dominées par un discours centré sur la rationalité économique et financière, reflet des idées du capitalisme global (Tienari et al., 2003) et de l’idéologie néolibérale (Fairclough, 2000).

Pour analyser les discours qui sont mobilisés dans le cas des activités internationales des FMN, nous avons appliqué une analyse critique de discours à un corpus de presse française. C'est cette analyse que nous allons maintenant présenter.

\section{Méthodologie de la recherche}

Cette recherche s'appuie sur le cas de Danone en Chine, dont la presse fait régulièrement écho, en particulier depuis 1996 et la prise de contrôle de Wahaha, l'une des plus grandes entreprises agroalimentaires chinoises. Ce cas renvoie à de multiples thèmes (modalités d'internationalisation, gestion des relations partenariales, échecs stratégiques, etc.), qui peuvent eux-mêmes donner lieu à différentes lectures. D’un point de vue stratégique par exemple, Danone réalise une importante part de son chiffre d'affaires en Chine $^{3}$ et fait de son développement dans le pays une priorité. D’un point de vue économique, le cas est aussi emblématique de la présence française en Chine, aujourd'hui reconnue comme un enjeu majeur (Allouche et al., 2008). Les activités de Danone en Chine présentent également une dimension politique, les relations partenariales entre la multinationale et différentes entreprises locales rejaillissant parfois sur les relations politico-diplomatiques franco-chinoises. Ces multiples "grilles de lectures», sans être exhaustives, montrent que le cas Danone est particulièrement représentatif du caractère protéiforme des activités internationales des FMN. Ce cas nous permettra ainsi d'observer quelles facettes des activités de la multinationale sont plus particulièrement mises en avant dans le discours médiatique, et surtout quelles interprétations en sont plus particulièrement données.

La constitution du corpus est un élément critique de l'ACD car elle détermine en grande partie la significativité des analyses (Leitch et Palmer, 2010). L'ACD n'impose aucune démarche spécifique ni ne prévoit un seuil en deçà duquel un corpus serait jugé trop pauvre. Dans les faits, le nombre de textes étudiés varie grandement, allant de textes uniques (Kuronen et al., 2005; Vaara et Tienari, 2008) à des corpus de plusieurs dizaines (Risberg et al., 2003; Tienari et al., 2003) voire plusieurs centaines de textes (Vaara et Tienari, 2002). Les choix méthodologiques en la matière impliquent un compromis entre rigueur et significativité (Alvesson et Karreman, 2000). Un nombre très restreint de textes peut être analysé plus finement, avec plus de rigueur, mais l'analyse perd alors en significativité.

Afin de trouver un compromis, nous avons choisi d'utiliser l'ensemble des articles relatifs aux activités de Danone en Chine dans trois des principaux titres de la presse économique française : Les Echos, La Tribune ${ }^{4}$, et Le Monde. Le nombre restreint de sources (et donc d'articles) permet une analyse plus fine et rigoureuse, tout en garantissant une certaine significativité des résultats puisqu'il s'agit de titres grand public avec une large diffusion, qui sont de fait susceptibles d'avoir une grande influence dans la sphère sociale. En outre, ces sources sont fréquemment reprises et citées dans d'autres contextes. Les articles ont été recherchés sur différentes bases de données, avec comme critère principal la présence des termes «Danone» et «Chine» ou «chinois(e)» dans leur titre. Seuls les articles d'une longueur supérieure à 200 mots ont ensuite été retenus, ce qui permet d'exclure les brèves, les articles rapportant des faits sans commentaires ni analyses, des données pauvres compte tenu de nos objectifs. La période initialement retenue pour la recherche allait de 2002 à 2013 mais aucun article antérieur à 2004 n’a été identifié. Si la présence de Danone en Chine est ancienne, la presse s'est surtout fait l'écho de cette présence au cours des dix dernières années car différents évènements ont marqué le développement de Danone dans le pays sur cette période (voir ci-après). Au total, le corpus est constitué de 65 articles d'une longueur moyenne de 456 mots (voir Annexe A).

Au-delà d'une simple analyse sémantique, l'ACD implique de porter attention aux détails linguistiques du texte et le repérage des différents discours nécessite de s'intéresser à la fois à qui s'exprime, à ce qui est exprimé et à la façon dont cela est fait, notamment au travers du champ lexical employé (Peltokorpi et Vaara, 2012). Nous avons donc choisi de mener une double analyse, thématique et lexicale. En d'autres termes, nous avons porté attention à la fois à ce qui est dit (analyse thématique) et à la façon dont cela est dit (analyse lexicale), ces deux aspects du discours participant à la construction et à la diffusion du sens ${ }^{5}$. Il s'agissait d'identifier à la fois les grands groupes d'idées présents dans le corpus (thèmes) et les grands groupes de mots utilisés pour les formuler (univers ou champs lexicaux). Notons également que l'analyse a été menée sur l'ensemble du corpus, et n'a révélé aucune différence notable entre les trois titres de presse considérés.

3. En 2012, la Chine a représenté $6 \%$ du chiffre d’affaires du groupe, ce qui place cette nation au $4 \mathrm{e}$ rang des pays où Danone est présent (Source : Document de référence Danone, 2012).

4. La Tribune ayant un temps été distribuée uniquement sous forme électronique, nous avons également inclus les articles tirés de «La tribune.fr».

5. La question du sujet (qui s’exprime?) n’a pas été explorée de façon aussi systématique. Nous avons considéré que les textes reflétaient avant tout le propos des journalistes. En effet, même quand des propos tenus par autrui y sont rapportés, ceux-ci sont réaménagés (coupes, reformulations, etc.) et s’accompagnent le plus souvent de commentaires, de telle sorte que le texte est finalement le fruit d'une subjectivité journalistique. Toutefois, lors du repérage des différents discours types dans le corpus, nous avons pu constater que certains discours s'appuyaient plus fréquemment que d’autres sur la citation de propos d’acteurs du terrain. Ce point est souligné dans la section consacrée aux résultats. 


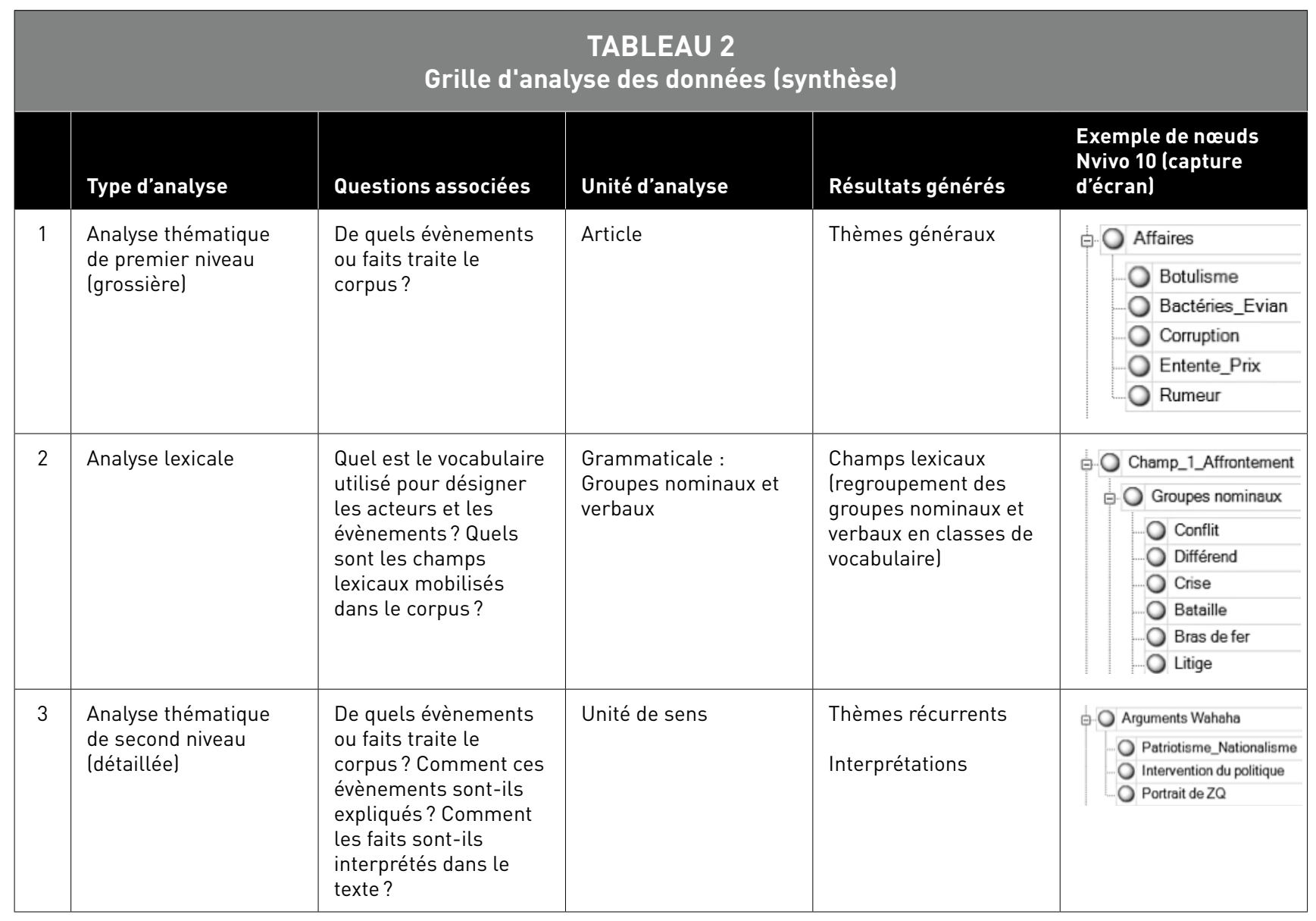

Cette double analyse a été menée à l'aide du logiciel Nvivo10 (Tableau 2). Nous avons encodé chaque article dans son intégralité, selon son sujet. Il s'agissait de voir quels évènements ou faits ont été plus particulièrement relayés dans la presse (Tableau 2, Ligne 1). A l'aide des fonctions de requête (fréquence de mots et recherche textuelle), nous avons mené l'analyse lexicale en identifiant les groupes grammaticaux se rapportant à des acteurs ou des faits. Ces mots ou expressions ont ensuite été regroupés en champs lexicaux (Tableau 2, Ligne 2 ). Nous avons également procédé à une analyse thématique $\mathrm{du}$ corpus, en identifiant des thèmes récurrents et en créant par regroupements successifs des catégories correspondantes (Tableau 2, Ligne 3).

Cette démarche implique une imbrication des types (thématique ou lexicale) et unités d'analyse (article, unité de sens ou unité grammaticale) (Tableau 2). Un même fragment de texte appartient ainsi à plusieurs catégories, ou "nœuds» dans Nvivo 10, ce qui permet d'exploiter la fonction de requête d'encodage matriciel du logiciel (Schéma 1). Cela permet par exemple de voir quels acteurs et quels types d'interprétations/ explications sont associés à chaque type d'évènement. Autre exemple, cela permet de voir, comment chaque évènement ou acteur est qualifié et comment ces différents qualificatifs (le vocabulaire utilisé) participent à la construction du sens.

Cette démarche renvoie à une approche inductive. Les nœuds, ont été générés de façon émergente, par «tâtonnement», en regroupant progressivement les unités d'analyse en champs (analyse lexicale) ou en thèmes (analyse thématiques) selon leurs similarités et leurs différences. Cela signifie que le retour vers la théorie, en particulier la typologie des discours présentée plus haut, n’a été opéré que dans un second temps. C'est alors un ensemble de "traces", tels que des champs lexicaux et des thèmes particuliers, qui permet de repérer les différents discours types dans le corpus (Vaara et Tienari, 2002). Les résultats ainsi générés ont une dimension subjective, et doivent ensuite faire l'objet de discussions et de critiques. Ils ne prétendent pas à la vérité et à l'exhaustivité, mais offrent des interprétations plausibles, cohérentes, quant aux différents discours et à leurs utilisations (Tienari et al., 2003).

\section{Résultats de la recherche}

D’une manière générale, le corpus traite du développement des activités de Danone en Chine. Lanalyse thématique révèle que quatre types de faits ou d'évènements ont été plus particulièrement relayés dans la presse : un conflit avec un partenaire local, les choix stratégiques du groupe, ses résultats sur le marché chinois, et différents scandales le touchant.

La majeure partie du corpus traite d'un conflit entre Danone et le groupe Wahaha, partenaires au sein de coentreprises créées en 1996 (36 articles). Dans ce conflit, qui s'est déroulé de 2007 à 2009, les deux partenaires s'accusaient mutuellement de ne pas respecter les termes de leur accord. 


\section{SCHÉMA 1}

\section{Exemple de matrice de croisement* (capture d'écran Nvivo10)}

\begin{tabular}{|c|c|c|c|c|c|}
\hline \multicolumn{6}{|l|}{ Requêtes } \\
\hline \multicolumn{6}{|c|}{ DIActeurs_Evènements - $\mathrm{A} \mathbf{x}$} \\
\hline & A: Affaires $\nabla$ & B : Conflit & $\nabla$ & C: Décisions_... $\nabla$ & D:Résultats $\nabla$ \\
\hline 1: Autre & \begin{tabular}{l|l}
5 & \\
\end{tabular} & 14 & & \begin{tabular}{l|}
31 \\
\end{tabular} & 1 \\
\hline 2: Cofco $\quad \nabla$ & 0 & 0 & & 6 & 0 \\
\hline 3:Danone $\nabla$ & 15 & 169 & & 77 & 30 \\
\hline 4: Dumex $\quad \nabla$ & 1 & 0 & & 0 & 0 \\
\hline 5:Emman... $\nabla$ & 0 & 11 & & 0 & 1 \\
\hline 6: Fonterra $\quad \nabla$ & 5 & 0 & & 0 & 0 \\
\hline 7:Franck ... $\quad \nabla$ & 0 & 4 & & 5 & 2 \\
\hline 8:Mengniu $\nabla$ & 0 & 0 & & 20 & 1 \\
\hline 9: Wahaha $\nabla$ & 4 & 114 & & 9 & 9 \\
\hline 10 : Zong Q... $\nabla$ & 0 & 91 & & 3 & 5 \\
\hline
\end{tabular}

* Cette matrice croise l'encodage par type d'évènement et l'encodage par acteur, tels qu'identifiés dans le corpus. Les valeurs correspondent au nombre de références d'encodage, qui peuvent ensuite être affichees en cliquant sur le contenu d'une cellule.

Selon Danone, son partenaire aurait illégalement développé des sociétés de distribution en concurrence directe avec leurs coentreprises. Selon Wahaha, d'une part ce montage avait été validé par Danone au moment de la conclusion de l'accord et, d'autre part, Danone aurait pris une part trop importante dans les coentreprises.

Le corpus traite également des décisions stratégiques de Danone sur le marché chinois (17 articles). Ces décisions relèvent de choix d'investissement et de désinvestissement ou encore de l'établissement de relations partenariales. Parmi celles-ci, les négociations avec le groupe laitier Mengniu, en vue d'établir un partenariat, sont celles qui ont reçu la plus grande couverture ( 8 articles). Après qu'un premier projet de partenariat ait été abandonné en 2007, les deux groupes ont annoncé en 2013 la création d'une coentreprise, détenue à $51 \%$ par Mengniu et $49 \%$ par Danone.

Deux dernières thématiques sont abordées par le corpus. Il s'agit, d'une part, de différentes affaires touchant de près ou de loin le groupe Danone (7 articles) et, d'autre part, de la présentation des résultats de Danone sur le marché chinois (5 articles). Les affaires ici évoquées sont de différentes natures. Il s'agit d'affaires sanitaires (une alerte au botulisme sur des produits laitiers, une alerte aux bactéries dans l'eau d'Evian, etc.) et d'affaires économiques, le groupe Danone ayant par exemple été mis en cause auprès d'autres groupes pour entente sur les prix dans le secteur du lait infantile.

\section{ACTEURS ET SCÉNARISATION DES ÉVÈNEMENTS : UNE LECTURE NATIONALISTE}

La façon dont les acteurs et les faits sont présentés dans les média sous-tend implicitement une lecture particulière des évènements, excluant de facto d'autres interprétations possibles de la réalité organisationnelle (Hellgren et al., 2002). En l'espèce, lorsque les média analysés évoquent les activités de Danone en Chine, ils décrivent un scénario où les acteurs ne sont pas seulement considérés pour eux-mêmes, mais aussi en tant que représentants de la nation dont ils sont issus. Cette interprétation est particulièrement patente dans le traitement du conflit opposant Danone et Wahaha. Si ces organisations sont souvent désignées par leur nom ${ }^{6}$, de nombreuses autres expressions sont utilisées. Celles-ci relèvent de quatre champs lexicaux distincts (Tableau 3).

Dans la majorité des cas, c'est un univers lexical relatif à la nation qui est mobilisé. Les organisations sont le plus souvent qualifiées selon leur pays d’origine. La mise en scène des évènements dans la presse véhicule ainsi une image où ce ne sont pas seulement deux organisations qui sopposent, mais également, à travers elles, deux pays. Ceci est d'autant plus explicite que l'ajout d'un qualificatif relevant de la nationalité est souvent répété au fil d'un même article. Dans de nombreux cas, il est aussi redondant, nétant pas strictement nécessaire à la compréhension du texte comme l'illustrent les quelques exemples suivants (Tableau 4).

6. Il s'agit le plus souvent de métonymies, où le nom de l'organisation se substitue à des personnes, typiquement les managers, ce qui est courant dans la façon de parler des organisations. 


\begin{tabular}{|c|c|c|c|c|c|c|c|c|c|c|c|c|c|c|c|}
\hline \multicolumn{16}{|c|}{$\begin{array}{c}\text { TABLEAU } 3 \\
\text { Champs lexicaux associés aux organisations impliquées dans le conflit Danone/Wahaha }\end{array}$} \\
\hline \multicolumn{4}{|c|}{ Champ 1 : Nation } & \multicolumn{4}{|c|}{ Champ 2 : Métier } & \multicolumn{4}{|c|}{ Champ 3 : Taille/Rang } & \multicolumn{4}{|c|}{ Champ 4 : Dirigeant } \\
\hline \multicolumn{4}{|c|}{$\begin{array}{l}\text { Exemples : «groupe français», } \\
\text { «groupe chinois», «société } \\
\text { hexagonale», «groupe } \\
\text { tricolore», «société chinoise» }\end{array}$} & \multicolumn{4}{|c|}{$\begin{array}{l}\text { Exemples : «groupe } \\
\text { alimentaire », «groupe } \\
\text { agroalimentaire», «spécialiste } \\
\text { des produits laitiers», } \\
\text { «producteur de boissons non } \\
\text { alcoolisées», «fabricant de } \\
\text { boissons» }\end{array}$} & \multicolumn{4}{|c|}{$\begin{array}{l}\text { Exemples : «géant», «leader», } \\
\text { «champion», «numéro un» }\end{array}$} & \multicolumn{4}{|c|}{$\begin{array}{l}\text { Exemples : «groupe fondé } \\
\text { et dirigé par Zong Qinghou», } \\
\text { «groupe de Zong Qinghou», } \\
\text { «groupe de Franck Riboud», } \\
\text { «groupe dirigé par Franck } \\
\text { Riboud» }\end{array}$} \\
\hline \multicolumn{2}{|c|}{ Danone* } & \multicolumn{2}{|c|}{ Wahaha } & \multicolumn{2}{|c|}{ Danone* } & \multicolumn{2}{|c|}{ Wahaha } & \multicolumn{2}{|c|}{ Danone* } & \multicolumn{2}{|c|}{ Wahaha } & \multicolumn{2}{|c|}{ Danone* } & \multicolumn{2}{|c|}{ Wahaha } \\
\hline Occ. & Sces. & Occ. & Sces. & Occ. & Sces. & Occ. & Sour. & Occ. & Sces. & Occ. & Sces. & Occ. & Sces. & Occ. & Sces \\
\hline 115 & 35 & 81 & 37 & 37 & 25 & 17 & 15 & 18 & 15 & 14 & 13 & 18 & 15 & 10 & 8 \\
\hline \multicolumn{4}{|c|}{ Total (sources) $=72$} & \multicolumn{4}{|c|}{ Total (sources) $=40$} & \multicolumn{4}{|c|}{ Total (sources) = 28} & \multicolumn{4}{|c|}{ Total (sources) $=23$} \\
\hline
\end{tabular}

* Recherche menée sur les seuls articles ayant pour sujet principal le conflit entre Danone et Wahaha

\begin{tabular}{|l|l|}
\hline \multicolumn{2}{|c|}{ Exemples d'utilisation de qualificatifs relevant de la nationalité } \\
\hline Expression & Utilisée pour \\
\hline Le groupe chinois Wahaha & Le groupe Wahaha \\
\hline Le chinois Hangzhou Wahaha Group & Hangzhou Wahaha Group \\
\hline Le groupe français Danone & Le groupe Danone \\
\hline
\end{tabular}

Cette lecture des évènements, nationale voire nationaliste (au sens de Vaara et Tienari, 2008), vaut également lorsque les relations entre organisations qui sont évoquées ne sont pas conflictuelles. Ainsi, les acteurs du partenariat Danone/ Mengniu sont-ils souvent qualifiés selon lors origine nationale (par exemple «le chinois Mengniu», «le leader chinois des yaourts», ou encore «le groupe tricolore»). Plus largement, lorsque le corpus évoque des relations entre différentes organisations, dans le cadre de la stratégie du groupe, on relève un usage quasi-systématique de qualificatifs relevant de la nationalité (par exemple : «numéro un chinois des produits laitiers » pour Bright Dairy ou «groupe chinois » pour China Hui Yuan Juice).

Parmi les différentes façons d'évoquer les organisations, on constate également que les média analysés recourent fréquemment à la personnification, en particulier concernant le groupe Wahaha. De nombreux passages mettent ainsi en opposition Danone d'une part, et Zong Qinghou, fondateur de Wahaha d'autre part (24 sources, 38 occurrences), mettant ainsi au même plan une organisation et un individu, comme l'illustrent ces exemples :

«Danone et Zong Qinghou, le fondateur de Wahaha, ont été partenaires [...] avant de se déchirer, publiquement depuis l'an dernier» (Tribune_4)

«Le conflit entre Danone et Zong Qinghou» (Monde_10)
Cette pratique discursive est elle aussi révélatrice d'une lecture nationaliste des évènements. En effet, le vocabulaire utilisé pour décrire Zong Qinghou emprunte pour une large part au champ de la nationalité (Champ 1 dans le Tableau 3), ce-dernier étant souvent qualifié, entre autres expressions, "d'homme d'affaires chinois», ou de "patron chinois». D'une manière générale, Zong Qinghou est présenté comme un individu emblématique de l'environnement d'affaires chinois :

«M. Zong est typique de ces patrons chinois dont l'ascension remonte aux prémices de l'ouverture économique, et qui ont su jouer des aides de l'Etat et de la restructuration des entreprises publiques pour fonder des groupes puissants. " (Monde_4)

\section{L'AFFRONTEMENT DE DEUX LOGIQUES}

La scénarisation des évènements liés au développement de Danone en Chine selon une lecture nationaliste va de pair avec la mise en contraste de deux logiques. Lanalyse thématique révèle que les comportements des acteurs sont restitués et justifiés de différentes manières et à travers différents discours, selon qu'ils sont français ou chinois.

\section{La logique de Danone}

La position de Danone dans le conflit l'opposant à Wahaha est avant tout légitimée par un discours reposant sur une logique 
légale. La principale revendication du groupe qui est mise en lumière par la presse porte sur les structures développées par son partenaire en marge de leurs coentreprises. Ces structures sont souvent qualifiées d' "illégales» ou d' «illégitimes». Ce jugement explicite est formulé en vertu d'un "contrat», d'un «accord» ou encore des «termes du partenariat» liant les deux organisations. Ce discours reprend en grande partie l'argumentaire du groupe français, s'appuyant fréquemment sur la citation de propos de cadres de Danone. Il est également souvent mis en relation avec les menaces de poursuites judiciaires de la part de la firme :
«Le groupe français d'agroalimentaire, estime pour sa part que les conditions de l'accord passé en 1996 ne sont pas respectées et a lancé aujourd'hui un recours judiciaire contre Zong Qinghou. Danone, qui détient $51 \%$ de la marque Wahaha, considère que son partenaire a violé leur accord...»(Tribune_22)

Si ce discours est utilisé pour justifier la position de Danone dans le conflit, celle-ci est aussi légitimée par un discours fondé sur une rationalité économique, semblable au discours rationaliste déjà identifié par d’autres (par exemple Vaara, 2002).

\begin{tabular}{|l|c|c|c|c|}
\hline \multicolumn{2}{|c|}{ Fréquence des termes associés à des grandeurs économiques } \\
\hline & \multicolumn{2}{|c|}{ Articles DS* } & \multicolumn{2}{c|}{ Reste du corpus } \\
\hline Termes** & Occ. & $\%$ & Occ. & 0,12 \\
\hline Chiffre d'affaires & 23 & 0,58 & 3 & 0,024 \\
\hline Part de marché & 10 & 0,25 & 27 & 0,22 \\
\hline Ventes & 15 & 0,38 & 9 & 0,07 \\
\hline Résultats & 6 & 0,15 & 3 & \\
\hline
\end{tabular}

* Articles ayant pour sujet principal les décisions stratégiques de Danone en Chine

** Les termes sont issus d'une analyse de fréquence sur l'ensemble du corpus. Aucune liste de mots n'a été définie a priori

\begin{tabular}{|c|c|c|c|c|}
\hline \multirow[b]{3}{*}{ Mot** } & \multirow{2}{*}{\multicolumn{2}{|c|}{$\begin{array}{r}\text { Champ lexical du mana } \\
\text { Articles DS* }\end{array}$}} & áquen & \\
\hline & & & \multicolumn{2}{|c|}{ Reste du corpus } \\
\hline & Occ. & $\%$ & Occ. & $\%$ \\
\hline activité(s) & 11 & 0,28 & 11 & 0,09 \\
\hline branche & 4 & 0,10 & 2 & 0,02 \\
\hline croissance & 10 & 0,25 & 16 & 0,13 \\
\hline développement & 9 & 0,23 & 10 & 0,08 \\
\hline développer & 8 & 0,20 & 7 & 0,06 \\
\hline investissement & 4 & 0,10 & 5 & 0,04 \\
\hline industrie & 6 & 0,15 & 2 & 0,02 \\
\hline marché(s) & 53 & 1,33 & 47 & 0,38 \\
\hline positionnement & 3 & 0,08 & 0 & 0,00 \\
\hline potentiel & 6 & 0,15 & 2 & 0,02 \\
\hline produit(s) & 65 & 1,63 & 61 & 0,49 \\
\hline secteur & 12 & 0,30 & 10 & 0,08 \\
\hline stratégie & 2 & 0,05 & 10 & 0,08 \\
\hline stratégique & 6 & 0,15 & 6 & 0,05 \\
\hline
\end{tabular}

* Articles ayant pour sujet principal les décisions stratégiques de Danone en Chine

** Les termes sont issus d'une analyse de fréquence sur l'ensemble du corpus. Aucune liste de mots n'a été définie a priori 
De nombreux passages mettent ainsi en avant les conséquences économiques de l'existence des structures «illégales» ou «illégitimes» pour Danone. De la même façon, la position de Danone est présentée comme relativement prudente. Laccent est mis sur la volonté de négocier et de parvenir à un compromis, les procédures judicaires ne constituant qu'un ultime recours face à une partie chinoise ne respectant pas les règles. Cette attitude prudente et raisonnable est le plus souvent légitimée par les conséquences économiques que représente une rupture du partenariat. Par exemple :

"Il refuse d'envenimer le conflit et de voir sa coentreprise, qui assure $70 \%$ de ses ventes en Chine, remise en question. » (Echo_21)

Le discours rationaliste ici identifié est également mobilisé dans le traitement médiatique d'autres faits, à savoir les décisions stratégiques du groupe. Cela transparait très clairement dans l'analyse lexicale. En menant une analyse de fréquence de mots comparée, on s'aperçoit notamment que les articles traitant de ce sujet recourent à des données chiffrées plus fréquemment que le reste du corpus. Les chiffres représentent ainsi plus de $6 \%$ du texte dans ces articles, contre 2,5\% par ailleurs. De même, les termes renvoyant à des grandeurs économiques sont plus présents (Tableau 5). On constate également que le champ lexical du management stratégique est plus fréquemment utilisé (Tableau 6).

\section{Logique des partenaires chinois}

Là où la presse évoque un raisonnement légal et économique dans le cas de Danone, une toute autre logique est mobilisée pour expliquer le comportement des organisations chinoises, et en particulier celui de Wahaha, qui est par ailleurs présenté comme plus déraisonnable et agressif que celui du groupe français. Les principaux arguments du groupe chinois reposent sur la négation de l'illégalité des sociétés hors des coentreprises, et l'identification de la menace que représente la position de Danone, le groupe français étant supposé vouloir prendre le contrôle de sociétés ne lui appartenant pas.

Au-delà de cette description factuelle, la position de Wahaha est expliquée comme relevant d'un patriotisme économique patent en Chine. Le pays serait ainsi saisi d'une "fièvre de patriotisme économique» (Echo_23). Ce patriotisme économique est également présenté comme un moyen de pression utilisé par Wahaha dans le conflit qui loppose à Danone. Ainsi, la position de Wahaha et de son dirigeant dans le conflit relèverait d'une instrumentalisation d'une certaine "fibre nationaliste», au service d'intérêts financiers et personnels. Cette interprétation de la position de Wahaha comme relevant d'une certaine rhétorique nationaliste reprend en partie les arguments de Danone :

«Wahaha avait tenté d'attiser le toujours très vif patriotisme économique chinois en associant l'entreprise de Franck Riboud "aux envahisseurs étrangers" voulant soumettre le pays.»(Echo_21)
"'M. Zong joue sur la fibre nationaliste, c'est une tactique', dit un proche du dossier côté français" (Monde_3)

Une autre interprétation de la position de Wahaha met en avant la dimension politique du conflit. Le dirigeant de Wahaha, aurait ainsi préféré « étouffer la dimension juridique de la crise pour lui donner une portée politique» (Echo_22). Cette seconde interprétation suggère que la position de Wahaha dans le conflit, n'est tenable que parce que son dirigeant bénéficie d'importants soutiens politiques :

"Les autorités de la province du Zhejiang [...] semblent décidées à soutenir le patron chinois dans toutes les arènes possibles et profitent de la perméabilité des institutions politiques et judiciaires pour peser sur certaines décisions clefs. »(Echo_16)

D’une manière générale, cette interprétation renvoie l'image d'une Chine où le politique et léconomique sont intimement liés. "Dans un pays comme la Chine, pas question de faire du business sans des appuis locaux, qui permettent de mieux appréhender les rouages du système» (Tribune_15). Cette imbrication entre politique et économique apparait également dans le portrait de Zong Qinghou dressé dans le corpus. Ce dernier est par exemple présenté comme «homme d'affaires devenu politicien»(Echo_16), et il est souligné combien «il entretient des liens étroits avec le pouvoir...» (Monde_12). De la même façon, les institutions judiciaires chinoises, sont parfois présentées comme partiales parce qu'elles seraient sous influence politique ${ }^{7}$ :

«S'il n'a jamais osé remettre en question la partialité des juges chinois, souvent liés aux pouvoirs politiques et économiques locaux, Danone espère faire valoir ses droits devant des cours étrangères plus impartiales. "(Echo_10)

\section{DANONE VICTIME...}

Les difficultés rencontrées par Danone dans ses activités en Chine sont le plus souvent interprétées à l'aune de cette image du pays. Danone est ainsi souvent présentée comme la victime d'un environnement d'affaires singulier. Ce positionnement de Danone en tant que victime est non seulement patent dans la couverture médiatique du conflit lopposant à Wahaha, mais il prévaut aussi lorsqu'il s'agit de rendre compte des différentes affaires touchant le groupe: "Autant d'affaires sans rapport apparent mais qui, toutes, risquent de pénaliser la marque Danone sur le marché chinois.» (Echo_1). Ainsi, ces affaires ne relèveraient-elles quasi-exclusivement que de tentatives, plus ou moins déguisées, de la part des autorités chinoises, de pénaliser les acteurs étrangers, dont Danone, au profit des industriels locaux, comme l'illustrent ces citations :

«Plusieurs analystes accusent les autorités chinoises de faire pression sur le groupe français en mettant en cause la qualité de ses eaux minérales. " (Monde_6)

"Des experts s'interrogent sur l'origine de la nouvelle affaire et se demandent si la rumeur n'aurait pas été encouragée, comme c'est régulièrement le cas en Chine, par des acteurs du

7. Dans le conflit avec Wahaha, les décisions juridiques en défaveur de Danone sont ainsi le plus souvent interprétées à l'aune de cette image. Cette interprétation reprend en grande partie l'argumentaire du groupe français, les propos d'acteurs de Danone étant même parfois cités. 
marché agacés par le récent succès des marques étrangères. »

(Echo_9)

La présentation des résultats de Danone sur le marché chinois met généralement en relief les conséquences des différentes affaires pour le groupe, qu'il s’agisse de ses résultats opérationnels ou de sa performance financière :

«Danone pénalisé par ses mésaventures en Chine. [...] Le groupe agroalimentaire revoit ses prévisions de résultats pour 2013. Danone subit l'impact de la fausse alerte sur son lait infantile.»(Echo_1)

\section{...OU DANONE CONQUÉRANT ?}

Si l'intervention du politique dans la vie économique et une certaine forme de nationalisme sont présentées comme deux caractéristiques de l'environnement des affaires chinois dont Danone est la victime, d'autres caractéristiques sont au contraire présentées comme sources d’opportunités. Ces particularités sociétales chinoises relèvent par exemple d'une certaine occidentalisation des habitudes de consommation :

"(Danone) a pris une participation de 22,18\% dans la société Hui Yuan [...] leader des boissons fruitées dans l'ex-empire du Milieu. Les jus de fruits sont promis à une très forte croissance en Chine. [...] Les Chinois, qui ne consommaient pas du tout de jus de fruits il y a vingt ans, se sont découvert une nouvelle passion, et cette industrie est promise à un formidable développement selon les experts. »

(Echo_24)

Dans l'ensemble, la Chine apparait ainsi comme une sorte d'Eldorado, un réservoir de croissance, en particulier par contraste avec les économies matures et les marchés saturés de l'Ouest. De ce point de vue, la présence française en Chine semble objectivement justifiée, présentant même un caractère inéluctable. Cette interprétation reprend en grande partie la rhétorique du groupe Danone :

«Le potentiel du marché chinois fait saliver Danone.» (Tribune_24)

"'Faut-il avoir peur de la Chine? Je ne crois pas, même s'il y a un risque [à y être présent], nous n'avons pas le choix, c'est le plus grand pays du monde et tout démarre ici', a expliqué

M. Riboud.» (Monde_1)

Les décisions stratégiques de Danone sont donc légitimées par des caractéristiques particulières du marché chinois que le groupe se devrait d'exploiter. Cette légitimation va de pair avec un recours massif au champ lexical du management stratégique tel que nous l'avons précédemment identifié.

\section{Discussion des résultats}

A ce jour, les rares travaux qui se sont intéressés au rôle des médias dans les processus stratégiques de sensemaking et sensegiving ont pour l'essentiel porté sur les F\&A (Hellgren et al., 2002; Kuronen et al., 2005; Zhu et McKenna, 2012). Cette recherche avait pour objectif d'élargir l'analyse, en se penchant sur la mise en sens des activités internationales des FMN. Au-delà de l'étude d'un contexte nouveau, ce travail aboutit à des résultats qui, par certains aspects, contrastent avec les recherches menées jusqu'alors. Nous abordons maintenant ce point, en mettant en perspective notre étude avec les recherches passées.

En premier lieu, notre recherche confirme que les média participent à la mise en sens de l'information stratégique, par le choix des sujets qu'ils traitent et l'interprétation qu'ils en donnent (Hellgren et al., 2002), participant ainsi à la construction sociale de la réalité (Risberg et al., 2003; Tienari et al., 2003). Parmi les multiples facettes des activités internationales des FMN, certaines sont ignorées et d'autres sont au contraire privilégiées. Le discours médiatique s'est essentiellement focalisé sur les difficultés rencontrées par Danone en Chine, d'autres sujets tels que le choix de son mode d'entrée et de ses partenaires ou encore l'impact sociétal de sa présence ayant été de facto marginalisés. Pourtant, si les difficultés de Danone en Chine sont indéniables, elles ne représentent qu'une facette des activités chinoises de la FMN. Sur la période retenue pour notre recherche (2002-2013), Danone a progressé sur plusieurs marchés, notamment celui des eaux et boissons, secteur pourtant touché directement par le conflit avec Wahaha. Ce secteur représente la première activité du groupe dans le pays, en termes de poids dans le chiffre d'affaires (Source: Rapport annuel, 2013). La part du chiffre d'affaires de Danone réalisée en Chine s'accroit régulièrement, plaçant cette nation au quatrième rang des pays où la FMN est présente, avec $7 \%$ des ventes du groupe . $^{8}$

Notre recherche confirme également l'intérêt de l'ACD et de l'usage des typologies de discours pour comprendre la façon dont les média rendent compte des activités et manœuvres stratégiques des entreprises. Comme les travaux sur le traitement médiatique des F\&A l'ont montré, il existe certains discours types (voir par exemple Vaara et Tienari, 2002). Dans cette recherche, nous avons observé que les discussions médiatiques sur les diverses activités internationales des FMN sarticulaient autour de quatre formes de discours imbriquées dans le texte: culturel/nationaliste, rationaliste, légaliste et individualiste (Tableau 7). Ces discours sont autant de cadres à travers lesquels le développement international d'une entreprise est interprété par les journalistes et dans le même temps expliqué à leur lectorat. En d'autres termes, ils constituent le canevas à travers lequel les média mettent en œuvre les processus de sensemaking et sensegiving autour de ces évènements.

Parmi l'ensemble de ces discours, les types culturel/ nationaliste, rationaliste, et individualiste ont déjà été identifiés par les précédents travaux mobilisant l'ACD dans le champ de la stratégie (Balogun et al., 2011; Vaara et Tienari, 2002; Vaara, 2002). En revanche un quatrième type, à ce jour ignoré, est apparu dans notre recherche. Il s'agit d'une forme de discours que l'on peut qualifier de «légaliste». Ce discours 


\begin{tabular}{|c|c|c|c|}
\hline Discours & Principales caractéristiques & Exemples & Utitisation \\
\hline $\begin{array}{l}\text { Culturel / } \\
\text { nationaliste }\end{array}$ & $\begin{array}{l}\text { - Définition des acteurs par leur } \\
\text { origine nationale } \\
\text { - Discours axé sur des } \\
\text { caractéristiques clés de } \\
\text { l'environnement des affaires } \\
\text { dans le pays lex : imbrication du } \\
\text { politique et de l'économique dans } \\
\text { le cas de la Chine) } \\
\text { - Opposition, confrontation entre } \\
\text { différents univers nationaux lex : } \\
\text { pensée en termes de France VS } \\
\text { Chinel }\end{array}$ & $\begin{array}{l}\text { Ensemble, les deux groupes vont } \\
\text { créer une coentreprise, détenue à } \\
51 \% \text { par le chinois et à } 49 \% \text { par le } \\
\text { français. (Monde_13) } \\
\text { Plus important sans doute le fait } \\
\text { que dans un pays comme la Chine, } \\
\text { pas question de faire du business } \\
\text { sans des appuis locaux, qui } \\
\text { permettent de mieux appréhender } \\
\text { les rouages du système. } \\
\text { (Tribune_15) }\end{array}$ & $\begin{array}{l}\text { - Justification des difficultés } \\
\text { rencontrées dans } \\
\text { l'internationalisation (conflit, } \\
\text { affaires) } \\
\text { - Spécificités nationales } \\
\text { comme motivation de } \\
\text { l'internationalisation... } \\
\text { - ...en articulation avec le } \\
\text { discours rationaliste (la Chine } \\
\text { comme Eldorado, réservoir de } \\
\text { croissance) }\end{array}$ \\
\hline Rationaliste & $\begin{array}{l}\text { - Discours fondé sur la rationalité } \\
\text { économique } \\
\text { - Discours objectivant } \\
\text { - Utilisation de données chiffrées } \\
\text { - Mobilisation du champ lexical du } \\
\text { management stratégique }\end{array}$ & $\begin{array}{l}\text { Ces annonces confirment aussi } \\
\text { la volonté de Danone de contrer } \\
\text { le ralentissement européen par } \\
\text { une montée en puissance sur } \\
\text { les marchés à forte croissance. } \\
\text { (Echo_5) } \\
\text { "Avec une faible consommation } \\
\text { par habitant, le marché offre un } \\
\text { important potentiel de croissance" } \\
\text { souligne Danone. (Tribune_24) }\end{array}$ & $\begin{array}{l}\text { - Légitimation des décisions } \\
\text { stratégiques liées à } \\
\text { l'internationalisation } \\
\text { (partenariats, cessions, etc.) } \\
\text { - Légitimation d'un positionnement } \\
\text { vis-à-vis d'un adversaire (conflit) } \\
\text { - ...en articulation avec le discours } \\
\text { légaliste }\end{array}$ \\
\hline Légaliste & $\begin{array}{l}\text { - Discours fondé sur une } \\
\text { rationalité légale } \\
\text { - Mobilisation du champ lexical } \\
\text { juridique }\end{array}$ & $\begin{array}{l}\text { Le groupe français a découvert } \\
\text { que son partenaire a illégalement } \\
\text { développé un réseau de production } \\
\text { et de vente de produits (Echo_22) } \\
\text { Le groupe français a envoyé } \\
\text { aujourd'hui un recours judiciaire } \\
\text { contre son partenaire chinois qu'il } \\
\text { accuse de rupture de contrat. } \\
\text { (Tribune_22) }\end{array}$ & $\begin{array}{l}\text { - Légitimation d'un positionnement } \\
\text { vis-à-vis d'un adversaire (conflit) } \\
\text { - ...en articulation avec le discours } \\
\text { rationaliste } \\
\text { - ...et culturel (ne s'applique qu'au } \\
\text { «camp» français) }\end{array}$ \\
\hline Individualiste & $\begin{array}{l}\text { - Personnification des } \\
\text { organisations et ou dénomination } \\
\text { des organisations en référence } \\
\text { aux dirigeants } \\
\text { - Application des caractéristiques } \\
\text { des dirigeants aux organisations }\end{array}$ & $\begin{array}{l}\text { Danone porte son différend avec } \\
\text { le chinois Zong Qinghou devant la } \\
\text { justice américaine (Tribune_17) } \\
\text { M. Zong est typique de ces patrons } \\
\text { chinois (Monde_4) }\end{array}$ & $\begin{array}{l}\text { - Légitimation d'un positionnement } \\
\text { vis-à-vis d'un adversaire (conflit) } \\
\text { - ...en articulation avec le discours } \\
\text { rationaliste } \\
\text { - ...et culturel (ne s'applique qu'au } \\
\text { «camp» français) }\end{array}$ \\
\hline
\end{tabular}

se focalise sur le caractère légal ou illégal des actions menées par les organisations. De par son caractère objectivant, il se rapproche du discours rationaliste, bien qu'il repose sur une rationalité légale plus quéconomique et managériale. Dans le corpus, ce discours est mobilisé pour légitimer les actions des organisations, et notamment la position de Danone face à Wahaha. Il s'articule alors avec le discours rationaliste, qui sert à souligner les conséquences économiques des pratiques «illégales» ou «illégitimes» de Wahaha pour Danone. Dans lensemble, la forme légaliste est moins présente que d'autres dans le corpus (voir paragraphe suivant) mais son identification complète toutefois les typologies issues de la littérature sur les F\&A. Elle suggère plus largement que celles-ci doivent être aménagées pour rendre compte de la construction discursive de la réalité organisationnelle dans d'autres contextes.

La principale divergence entre les travaux ayant mobilisé l'ACD dans le contexte des F\&A et notre propre recherche porte sur la prédominance de certains discours dans le texte. Comme nous l'avons rappelé, les discussions autour des activités et manœuvres stratégiques des entreprises sont habituellement dominées par un discours rationaliste (Vaara, 2002; Vaara et Tienari, 2002). Si le discours culturel/ nationaliste est présent, il n'est le plus souvent considéré que comme une alternative ou une voie de résistance à une idéologie néolibérale dominante (Hellgren et al., 2002; Kuronen et al., 2005; Risberg et al., 2003). Nos analyses du discours médiatique montrent au contraire que, dans le cas de Danone en Chine, ce discours est dominant. Les entreprises concernées sont le plus souvent associées à leur origine nationale (par exemple «le Français» (Danone) vs. «le Chinois» (Wahaha)) et les comportements des organisations sont souvent justifiés par des caractéristiques nationales distinctes. Par exemple, la position de Wahaha dans le conflit lopposant à Danone est présentée comme le résultat d'un certain patriotisme économique et d'une imbrication entre le politique et léconomique, supposés être des particularités de l'environnement d'affaires chinois. 
Le discours culturel/nationaliste est également dominant parce que les autres formes de discours lui sont subordonnées. Par exemple, si le discours rationaliste est bien présent dans le corpus, il ne prend sens qu'en rapport avec le discours culturel/ nationaliste. Quand les décisions stratégiques de Danone sont légitimées, elles le sont en premier lieu par des particularités chinoises. Il ne s'agirait que de réactions logiques et rationnelles aux évolutions sociétales et économiques du pays. Le discours individualiste est lui aussi présent, focalisé sur les dirigeants des entreprises mais, comme le discours rationaliste, il est subordonné au discours culturel/nationaliste. Par exemple, le portrait de Zong Qinghou, fondateur et PDG du groupe Wahaha, souvent présenté comme le principal voire le seul interlocuteur de Danone en Chine, est ainsi celui d'un Chinois «typique».

Ces résultats montrent que les schémas discursifs utilisés par les média dépendent largement des évènements qu'ils traitent et de leur contexte. Si l'usage d'un discours rationaliste semble s'imposer pour justifier certaines manœuvres stratégiques telles que des F\&A (Hellgren et al., 2002; Vaara et Tienari, 2002), un autre schéma discursif est privilégié lorsqu'il s'agit de rendre compte du développement international d'une FMN et à plus forte raison des difficultés qu'il suscite. Ce constat soulève la question des raisons pour lesquelles le schéma alors privilégié est celui du discours culturel/nationaliste. Deux pistes explicatives peuvent ici être avancées.

La prédominance de cette lecture des évènements peut d'abord être vue comme une forme de biais cognitif. Il est en effet très probable que les processus de sensemaking et sensegiving médiatiques, en tant qu'activités impliquant un jugement, soient sujets à de multiples distorsions. Il peut s'agir notamment d'une forme de biais d'auto-complaisance (Feather et Simon, 1971), les média français attribuant volontiers les difficultés de Danone en Chine à une cause externe, préservant par là même une image positive du groupe hexagonal. Le discours rationaliste, qui prévaut habituellement dans les discussions autour des manœuvres stratégiques des entreprises devient alors secondaire, suggérant que Danone est principalement victime d'un environnement local particulièrement difficile. D'autres explications alternatives (ou complémentaires) qui souligneraient la responsabilité du groupe, à travers ses décisions stratégiques (choix d'un partenaire, modalités de coopération, etc.) sont dans le même temps marginalisées. Ce biais d'auto-complaisance serait alors lui-même lié à un phénomène de cadrage (Tversky et Kahneman, 1981). Le fait de penser les activités d'une FMN dans un de ses marchés hôtes en termes de «déboires» ou encore de «mésaventures» amènerait plus particulièrement les journalistes à développer une argumentation sur les causes de ces problèmes.

Une autre explication de la prédominance du discours culturel/nationaliste tient, en l'occurrence, aux origines de ce discours. En effet, un discours napparait jamais ex nihilo. Les structures discursives et narratives existantes influencent l'émergence de nouvelles interprétations (Balogun et al., 2014) et si certaines interprétations en viennent à être communément admises c'est souvent quelles entrent en résonance avec des discours plus larges, qui contribuent à leur crédibilité narrative (Barry et Elmes, 1997). Nous émettons donc ici l'hypothèse que les processus de sensemaking et sensigiving médiatiques sont influencés par une représentation particulière de la Chine, notamment issue de la littérature managériale (Fernandez, 2003, 2007). Cette littérature, largement diffusée et popularisée par les experts que les média consultent, insiste sur l'altérité culturelle de la Chine et s'attache à démontrer "qu'une 'approche culturelle de l'économie chinoise' s'impose, réalisant que nos propres grilles de lecture seraient insuffisantes pour comprendre la Chine» (Fernandez, 2007, p. 1). Il n'est donc pas surprenant que les média conçoivent et présentent la Chine comme un pays radicalement autre, marqué par une différence culturelle problématique dans le développement des activités commerciales avec les étrangers. Cette image, qui imprègne l'imaginaire collectif occidental, servirait alors de grille de lecture implicite aux média lorsqu'ils traitent du développement de FMN occidentales en Chine. Par exemple, les liens étroits entre acteurs issus des sphères économique et politique, présentés comme un élément déterminant des difficultés de Danone en Chine, peuvent être rapprochés (sans que le concept ne soit explicitement cité dans la presse) de la notion de guanxi, abondamment développée dans la littérature managériale (Yang, 1994). Cette littérature fait des relations interpersonnelles et du réseautage avec échange mutuel de faveurs un fondement des affaires en Chine. De la même façon, le patriotisme chinois est une notion récurrente dans cette littérature, associée à celle de nationalisme, de sinité, de valeurs chinoises et à l'existence supposée d'un management ou capitalisme chinois (Dirlik, 1997). Comme le remarquent Risberg et al. (2003), l'usage de tels lieux communs constitue une façon banale de (re)construire ou de faire appel à des identités, des représentations nationales collectives qui séduisent le lecteur.

\section{Conclusion}

Au cours de cette recherche, nous nous sommes efforcés de comprendre comment la presse du pays dorigine faisait sens des activités menées par les FMN dans leurs marchés hôtes. Nous avons analysé les discours que la presse utilise pour rendre compte des activités de Danone en Chine et identifié parmi ceux-ci un type encore à ce jour ignoré, à savoir le discours légaliste. Nous avons également montré que le discours culturel/nationaliste est dominant, contrairement aux recherches existantes selon lesquelles le discours rationaliste prévaut le plus souvent. Cette recherche renseigne ainsi sur les pratiques des média lorsqu'ils font sens de l'information stratégique, suggérant que cette mise en sens implique des biais cognitifs et mobilise des grilles de lectures latentes (ici culturaliste). Elle ouvre un questionnement sur la façon dont ces interprétations sont à leur tour intégrées et réinterprétées par les stratèges. En effet, les interprétations singulières qui se construisent dans la presse économique sont susceptibles par leur large diffusion d'influencer la conception de la stratégie et dêtre prises en compte dans les choix d'internationalisation des FMN. Le discours médiatique, comme toute autre forme de discours, présente ainsi vraisemblablement un certain 
pouvoir performatif (Balogun et al., 2014) qu'il conviendrait d'étudier plus en détail.

Cette recherche comporte certaines limites qui tiennent à la singularité du cas étudié et qui constituent autant de pistes de recherche. On peut en effet se demander si les activités d'autres entreprises françaises en Chine sont interprétées de la même façon par les média. De même, dans quelle mesure aurions-nous obtenu les mêmes résultats s'il sétait agi des activités de Danone dans un autre pays que la Chine? Les média d'autres pays traitent ils des activités de leurs multinationales de la même façon? Ces questions interrogent toutes les conditions sous lesquelles un discours type en vient à s'imposer comme grille de lecture principale.

\section{Références}

Abrahamson, E. (1996). «Management fashion», Academy of Management Review, Vol. 21, N 1, p. 254-285.

Allouche, J.; Domenach, J.L.; FROISSART, C.; GILBERT, P.; BOULAIRE, M. LE (2008). «Les entreprises françaises en Chine. Environnement politique, enjeux socioéconomiques et pratiques managériales ", Les études du CERI, N 145-146.

Alvesson, M.; Karreman, D. (2000). «Varieties of discourse : on the study of organizations through discourse analysis ", Human Relations, Vol. 53, № 9, p. 1125-1149.

Balogun, J.; Jacobs, C.; Jarzabkowski, P.; Mantere, S.; VaAra, E. (2014). «Placing strategy discourse in context : sociomateriality, sensemaking, and power : placing strategy discourse in context», Journal of Management Studies, Vol. 51, $\mathrm{N}^{\circ}$ 2, p. $175-201$.

Balogun, J.; Jarzabkowski, P.; VaAra, E. (2011). "Selling, resistance and reconciliation : A critical DISCURSIVE approach to subsidiary role evolution in MNEs », Journal of International Business Studies, Vol. 42, N 6, p. 765-786.

BARrY, D.; Elmes, M. (1997). «Strategy retold : toward a narrative view of strategic discourse. ", Academy of Management Review, Vol. 22, $\mathrm{N}^{\circ}$ 2, p. 429-452.

Chanal, V.; Tannery, F. (2005). «La communication de la stratégie ou l'art de persuader. Le cas du groupe Lafarge», Revue française de gestion, Vol. 31, $\mathrm{N}^{\circ}$ 159, p. 165-188.

Chanal, V.; Tannery, F. (2007). «La rhétorique de la stratégie : comment le dirigeant crée-t-il un ordre pour l'action », Finance, contrôle, stratégie, Vol. 10, $\mathrm{N}^{\circ}$ 2, p. 97.

DiJK, T.A. VAN (1993). «Principles of critical discourse analysis. », Discourse \& Society, Vol. 4, N² 2, p. 249-283.

Dirlik, A. (1997). "Critical reflections on "Chinese capitalism” as a paradigm», dans Brown R.A. (éditeur), Chinese Business Enterprise, Londres, Routledge, p. 17-38.

Ezzamel, M.; Willmott, H. (2008). «Strategy as Discourse in a Global Retailer : A Supplement to Rationalist and Interpretive Accounts», Organization Studies, Vol. 29, N² 2, p. 191-217.

Fairclough, N. (2000). "Language and neo-liberalism», Discourse \& Society, Vol. 11, N² 2, p. 147-148.

FAIRCLOUGH, N. (2005). «Peripheral vision : discourse analysis in organization studies : the case for critical realism", Organization Studies, Vol. 26, Nº 6, p. 915-939.
Feather, N.T.; Simon, J.G. (1971). «Attribution of responsibility and valence of outcome in relation to initial confidence and success and failure of self and other", Journal of Personality and Social Psychology, Vol. 18, $\mathrm{N}^{\circ}$ 2, p. 173-188.

Fernandez, B. (2003). «Confiance et méfiance dans le rapport à l'altérité asiatique : le cas de l'expérience d'expatriés français en Chine», dans Thuderoz C., Mangematin V. (éditeurs), Des Mondes de confiance, Editions CNRS.

Fernandez, B. (2007). "Management interculturel en Chine, enjeux et perspectives ", Actes du 3ème congrès du Réseau Asie, Paris, IMASIE

Gioia, D.A.; Chittipeddi, K. (1991). «Sensemaking and sensegiving in strategic change initiation ", Strategic Management Journal, Vol. 12, $\mathrm{N}^{\circ}$ 6, p. 433-448.

Hellgren, B.; Löwstedt, J.; Puttonen, L.; Tienari, J.; VaAra, E.; Werr, A. (2002). "How issues become (re)constructed in the media : discursive practices in the Astrazeneca merger.", British Journal of Management, Vol. 13, N 2, p. 123.

Kuronen, M.-L.; Tienari, J.; VaAra, E. (2005). «The merger storm recognizes no borders : an analysis of media rhetoric on a business manoeuvre», Organization, Vol. 12, N² 2, p. 247-273.

Leitch, S.; Palmer, I. (2010). «Analysing texts in context : current practices and new protocols for critical discourse analysis in organization studies ", Journal of Management Studies, Vol. $47, \mathrm{~N}^{\circ} 6$, p. $1194-1212$.

Maitlis, S.; Lawrence, T.B. (2007). «Triggers and enablers of sensegiving in organizations. ", Academy of Management Journal, Vol. 50, $\mathrm{N}^{\circ} 1$, p. 57-84.

Mazza, C.; Alvarez, J.L. (2000). «Haute couture and pret-a-porter : the popular press and the diffusion of management practices », Organization Studies, Vol. 21, N³ 3. p67-588.

Paroutis, S.; Heracleous, L. (2013). «Discourse revisited : Dimensions and employment of first-order strategy discourse during institutional adoption », Strategic Management Journal, Vol. 34, $\mathrm{N}^{\circ}$ 8, p. 935-956.

Peltokorpi, V.; VaAra, E. (2012). «Language policies and practices in wholly owned foreign subsidiaries : A recontextualization perspective», Journal of International Business Studies, Vol. 43, $\mathrm{N}^{\circ}$ 9, p. 808-833.

Phillips, N.; Sewell, G.; Jaynes, S. (2008). «Applying critical discourse analysis in strategic management research", Organizational Research Methods, Vol. 11, N 4, p. 770-789.

RIAD, S.; VAARA, E. (2011). "Varieties of national metonymy in media accounts of international mergers and acquisitions. ", Journal of Management Studies, Vol. 48, N 4, p. 737-771.

Risberg, A.; Tienari, J.; VaAra, E. (2003). «Making sense of a transnational merger : media texts and the (re)construction of power relations.», Culture \& Organization, Vol. 9, № 2, p. 121.

Rouleau, L. (2005). "Micro-practices of strategic sensemaking and sensegiving : how middle managers interpret and sell change every day», Journal of Management Studies, Vol. 42, $\mathrm{N}^{\circ}$ 7, p. 1413-1441.

Rouleau, L.; Balogun, J. (2011). «Middle managers, strategic sensemaking, and discursive competence : middle managers and strategic sensemaking", Journal of Management Studies, Vol. 48, No 5, p. 953-983. 
SAMra-Fredericks, D. (2003). «Strategizing as lived experience and strategists' everyday efforts to shape strategic direction", Journal of Management Studies, Vol. 40, N 1, p. 141-174.

Sergot, B.; Claret, N. (2011). «La légitimation discursive des stratégies de localisation à l'international : une étude de cas comparée de deux groupes agroalimentaires français", Management international, Vol. 16, $\mathrm{N}^{\circ} 1$, p. 45.

Tienari, J.; VAara, E.; BJÖrkman, I. (2003). «Global capitalism meets national spirit discourses in media texts on a cross-border acquisition », Journal of Management Inquiry, Vol. 12, $\mathrm{N}^{\circ} 4$, p. 377-393.

Tversky, A.; Kahneman, D. (1981). «The framing of decisions and the psychology of choice», Science, Vol. 211, $\mathrm{N}^{\circ} 4481$, p. $453-458$.

VAARA, E. (2002). «On the discursive construction of success/failure in narratives of post-merger integration. ", Organization Studies, Vol. 23, N 2, p. 211-248.
VAara, E.; Tienari, J. (2002). «Justification, legitimization and naturalization of mergers and acquisitions : a critical discourse analysis of media texts », Organization, Vol. 9, $\mathrm{N}^{\circ}$ 2, p. 275-304.

VaAra, E.; Tienari, J. (2008). «A discursive perspective on legitimation strategies in multinational corporations", Academy of Management Review, Vol. 33, № 4, p. 985-993.

YANG, M.M. (1994). Gifts, favors and banquets : The art of social relationships in China, Ithaca, Cornell University Press.

Zhu, Y.; Mckenna, B. (2012). «Legitimating a Chinese takeover of an Australian iconic firm : revisiting models of media discourse of legitimacy», Discourse \& Society, Vol. 23, $\mathrm{N}^{\circ}$, p. 525-552.

\section{ANNEXE A : Détail du corpus}

ETIQUETTE TITRE DE L'ARTICLE

\begin{tabular}{|c|c|c|c|}
\hline Echo_1 & Danone pénalisé par ses mésaventures en Chine & Les Echos & $17 / 10 / 13$ \\
\hline Echo_2 & Danone accusé par la télévision d’Etat chinoise & Les Echos & $17 / 09 / 13$ \\
\hline Echo_3 & Danone forcé de rappeler du lait infantile en Chine & Les Echos & $06 / 08 / 13$ \\
\hline Echo_4 & Danone et Nestlé mis en cause par la Chine pour entente sur les prix & Les Echos & 03/07/13 \\
\hline Echo_5 & Danone repart à l'offensive pour vendre ses yaourts en Chine & Les Echos & $21 / 05 / 13$ \\
\hline Echo_6 & Danone à la peine sur le yaourt standard en Chine & Les Echos & $09 / 12 / 11$ \\
\hline Echo_7 & Eau purifiée : Danone se désengage en Chine & Les Echos & $15 / 07 / 11$ \\
\hline Echo_8 & Comment le litige avec Wahaha a fortifié Danone sur le marché de l'eau en Chine & Les Echos & $29 / 09 / 10$ \\
\hline Echo_9 & Danone malmené par une rumeur en Chine & Les Echos & $13 / 02 / 09$ \\
\hline Echo_10 & Le conflit commercial entre Danone et son grand associé chinois s'enlise & Les Echos & $16 / 01 / 09$ \\
\hline Echo_11 & Danone prêt à rompre ses liens avec sa coentreprise chinoise Wahaha & Les Echos & $22 / 05 / 08$ \\
\hline Echo_12 & Ultime bras de fer entre Danone et son partenaire chinois Wahaha & Les Echos & $14 / 04 / 08$ \\
\hline Echo_13 & Danone fait un geste pour calmer le jeu avec le chinois Wahaha & Les Echos & $18 / 01 / 08$ \\
\hline Echo_14 & Encore un revers pour Danone en Chine & Les Echos & $19 / 12 / 07$ \\
\hline Echo_15 & Danone propose un armistice à son partenaire chinois Zong Qinghou & Les Echos & $17 / 12 / 07$ \\
\hline Echo_16 & En Chine, Danone perd une manche contre son partenaire Zong Qinghou & Les Echos & $11 / 12 / 07$ \\
\hline Echo_17 & Danone vend ses parts dans le laitier chinois Bright Dairy & Les Echos & $16 / 10 / 07$ \\
\hline Echo_18 & Affaire Danone : le chinois Wahaha contre-attaque en justice & Les Echos & $14 / 06 / 07$ \\
\hline Echo_19 & Le groupe Danone exclut de se retirer de sa coentreprise chinoise & Les Echos & 13/06/07 \\
\hline Echo_20 & Danone obtient le départ de son turbulent partenaire chinois & Les Echos & 08/06/07 \\
\hline Echo_21 & Danone poursuit son partenaire chinois en justice & Les Echos & 10/05/07 \\
\hline Echo_22 & Danone lance un ultimatum à son turbulent partenaire chinois & Les Echos & $12 / 04 / 07$ \\
\hline Echo_23 & Danone aux prises avec le patriotisme économique chinois & Les Echos & $11 / 04 / 07$ \\
\hline Echo_24 & Le groupe Danone investit dans le jus de fruits en Chine & Les Echos & 04/07/06 \\
\hline Echo_25 & Danone dope sa recherche dans les produits laitiers en Chine & Les Echos & $02 / 11 / 05$ \\
\hline Monde_1 & Comment Danone espère faire manger ses biscuits et ses yaourts aux Chinois & Le Monde & $09 / 11 / 04$ \\
\hline
\end{tabular}




\begin{tabular}{|c|c|c|c|}
\hline Monde_2 & Danone, cible du patriotisme économique chinois & Le Monde & 10/04/07 \\
\hline Monde_3 & Wahaha surfe sur le patriotisme économique chinois pour dénoncer son partenaire Danone & Le Monde & $12 / 04 / 07$ \\
\hline Monde_4 & En Chine, Danone se heurte aux ambiguïtés du capitalisme d'Etat & Le Monde & 13/04/07 \\
\hline Monde_5 & Danone engage des poursuites contre son partenaire chinois Wahaha & Le Monde & $11 / 05 / 07$ \\
\hline Monde_6 & Frictions sur l'eau d'Evian entre Danone et la Chine & Le Monde & $30 / 05 / 07$ \\
\hline Monde_7 & Ce milliardaire chinois qui défie Danone & Le Monde & 05/07/07 \\
\hline Monde_8 & Danone pénalisé par son conflit avec le chinois Wahaha & Le Monde & $01 / 08 / 07$ \\
\hline Monde_9 & Danone rompt sa coentreprise en Chine avec Mengniu & Le Monde & $20 / 12 / 07$ \\
\hline Monde_10 & Danone s'associe avec Weight Watchers sur le marché chinois & Le Monde & $07 / 02 / 08$ \\
\hline Monde_11 & Danone a les mains libres en Inde, mais pas en Chine & Le Monde & 16/04/09 \\
\hline Monde_12 & $\begin{array}{l}\text { En Chine, Danone finit par vendre sa coentreprise avec Wahaha pour ne pas compromettre } \\
\text { son développement }\end{array}$ & Le Monde & $01 / 10 / 09$ \\
\hline Monde_13 & Danone se réattaque au marché chinois du yaourt & Le Monde & $20 / 05 / 13$ \\
\hline Tribune_1 & Agro-alimentaire; Danone touché par un nouveau scandale laitier en Chine & La Tribune & $06 / 08 / 13$ \\
\hline Tribune_2 & Danone se renforce en Chine en nouant une nouvelle alliance & LaTribune.fr & $20 / 05 / 13$ \\
\hline Tribune_3 & Danone et Wahaha se séparent à l'amiable en Chine & La Tribune & $01 / 10 / 09$ \\
\hline Tribune_4 & Conflit Danone/Wahaha : la justice tranche à nouveau en faveur du chinois & LaTribune.fr & 05/08/08 \\
\hline Tribune_5 & Danone choisit de se développer sans Mengniu en Chine & LaTribune.fr & 18/12/07 \\
\hline Tribune_6 & agroalimentaire; Danone tente de solder à l'amiable son différend avec le chinois Wahaha & La Tribune & $17 / 12 / 07$ \\
\hline Tribune_7 & Danone souhaite négocier avec son ex-partenaire chinois & LaTribune.fr & $14 / 12 / 07$ \\
\hline Tribune_8 & Danone fait appel de la décision du tribunal chinois qui lui a donné tort & LaTribune.fr & $11 / 12 / 07$ \\
\hline Tribune_9 & Un tribunal chinois donne tort à Danone & LaTribune.fr & 10/12/07 \\
\hline Tribune_10 & Danone gagne une manche dans la bataille avec le chinois Wahaha & LaTribune.fr & $21 / 11 / 07$ \\
\hline Tribune_11 & Danone : la Bourse s'inquiète des problèmes chinois & LaTribune.fr & $31 / 07 / 07$ \\
\hline Tribune_12 & Danone porte plainte contre son partenaire chinois Wahaha & LaTribune.fr & $12 / 07 / 07$ \\
\hline Tribune_13 & Danone : Wahaha obtient un arbitrage chinois & LaTribune.fr & $18 / 06 / 07$ \\
\hline Tribune_14 & agroalimentaire; Le problème chinois de Danone serait surestimé & La Tribune & $14 / 06 / 07$ \\
\hline Tribune_15 & Le conflit avec son partenaire chinois dans Wahaha pourrait fragiliser Danone & LaTribune.fr & 08/06/07 \\
\hline Tribune_16 & Danone annonce le départ du président de la JV Wahaha en Chine & LaTribune.fr & 07/06/07 \\
\hline Tribune_17 & Danone porte son différend avec le chinois Zong Qinghou devant la justice américaine & LaTribune.fr & 05/06/07 \\
\hline Tribune_18 & agroalimentaire; Nouvelles difficultés pour Danone en Chine & La Tribune & $31 / 05 / 07$ \\
\hline Tribune_19 & Danone sanctionné en Bourse après un nouveau problème en Chine & LaTribune.fr & $30 / 05 / 07$ \\
\hline Tribune_20 & Danone engage des poursuites judiciaires face à son partenaire chinois Wahaha & LaTribune.fr & $09 / 05 / 07$ \\
\hline Tribune_21 & Après la Chine, Danone confronté à des difficultés avec son partenaire en Inde & LaTribune.fr & $13 / 04 / 07$ \\
\hline Tribune_22 & Le ton monte d'un cran entre Danone et son partenaire chinois Wahaha & LaTribune.fr & $11 / 04 / 07$ \\
\hline Tribune_23 & agroalimentaire; Danone se heurte au nationalisme chinois & La Tribune & $11 / 04 / 07$ \\
\hline Tribune_24 & Le potentiel du marché chinois fait saliver Danone & La Tribune & $19 / 12 / 06$ \\
\hline Tribune_25 & Danone passe à l'offensive sur le marché des produits laitiers en Chine & LaTribune.fr & $18 / 12 / 06$ \\
\hline Tribune_26 & Danone se renforce en Chine & La Tribune & $04 / 07 / 06$ \\
\hline Tribune_27 & Danone veut convertir les Chinois aux yaourts & La Tribune & $02 / 11 / 04$ \\
\hline
\end{tabular}

